\title{
CoviD-19 Collaborative working on a 7-day rota reduces length of stay
}

\author{
Authors: Louis ] Koizia, ${ }^{\mathrm{A}}$ Melanie Dani, ${ }^{\mathrm{A}}$ Ganan Sritharan, ${ }^{\mathrm{A}}$ George Peck ${ }^{\mathrm{A}}$ and Michael B Fertleman ${ }^{\mathrm{A}}$
}

\section{A 7-day consultant-geriatrician-led service across five surgical wards, with integrated working among surgeons and physicians, was implemented in response to the COVID-19 pandemic. Our model has shown to increase discharge rates and improve MDT wellbeing. Embedded physician working with surgeons could be key in the recovery to COVID-19.}

KEYWORDS: COVID-19, surgical physicians, 7-day working, geriatrics

DOI:10.7861/fhj.2020-0142

\section{Background}

Following the declaration of the severe acute respiratory coronavirus 2019 (COVID-19) pandemic in March 2020, we were concerned about increased demand for medical beds in our hospital. Our solution was to adopt a 7-day consultant-geriatrician-led service to manage the five surgical wards (totalling 120 beds) alongside our surgical colleagues.

Previously, 7-day consultant working has been shown to reduce mortality and length of stay. Additionally, integration of physicians within surgical teams has been shown to reduce length of stay and hospital-acquired complications. ${ }^{2}$

\section{Model}

Prior to the pandemic, the Trust employed four surgical physicians, geriatricians by background, who worked with orthopaedics, major trauma, vascular and general surgery to provide support for medically complex and elderly patients.

As a result of the COVID-19 pandemic and in anticipation of increasing numbers of patients, the Trust increased the number of consultant physicians working in surgery, from four to eight (three emergency locum contracts and one transfer from a non-emergency site). In our model, surgeons managed the surgical patients on these wards. The physicians provided 7-day care for patients with COVID-19 and ensured early and appropriate escalation decisions for both medical and surgical patients. The physicians also led daily multidisciplinary board rounds for both medical and surgical patients to ensure smooth and timely decisions.

Author: ${ }^{A}$ consultant geriatrician, Cutrale Perioperative and Ageing Group, Imperial College London, London, UK

\section{Results and discussion}

Following implementation of our model, surgical discharges increased by $50 \%$, and there was a $27 \%$ greater weekly discharge rate among medical patient compared with wards run by other physicians. In addition, following surveys of the multidisciplinary team (MDT), the impact of the new model led to improved wellbeing among the junior doctors and ensured effective and timely MDT communication.

The integrated working among surgeons and physicians shows that an all-age 7-day model of care by a physician embedded in surgery has great potential. During the recovery phase of COVID-19, we anticipate that our model will improve flow on surgical wards, resulting in fewer cancellations and reduced waiting times.

\section{Conclusion}

We believe that embedding physicians working with surgeons on a 7-day rota improves patient flow through the hospital and will be essential for future periods of increased demand.

\section{Acknowledgements}

The authors would like to thank Catherine Urch, Martina Dinneen and Ruth Dixon Del Tufo for their support.

\section{References}

1 Leong K, Titman A, Brown M et al. A retrospective study of seven-day consultant working: reductions in mortality and length of stay. J R Coll Physicians Edinb 2015;45:261-7.

2 Shipway D, Koizia L, Winterkorn $N$ et al. Embedded geriatric surgical liaison is associated with reduced inpatient length of stay in older patients admitted for gastrointestinal surgery. Future Healthc ] 2018:5:108-16.

Address for correspondence: Louis J Koizia, Cutrale Perioperative and Ageing Group, Imperial College London, White City Campus, London W12 0BZ, UK. Email: louis.koizia05@imperial.ac.uk 